\title{
SUPLEMENTAÇÃO DE SELÊNIO NA DIETA E QUALIDADE ESPERMÁTICA DO SÊMEN DE TOUROS BRANGUS
}

\section{DIETARY SELENIUM SUPPLEMENTATION AND SPERM QUALITY IN BRANGUS BULLS SEMEN}

\author{
Pedro Paulo Tsuneda ${ }^{1 *}$ ORCID - http://orcid.org/0000-0001-8508-7931 \\ Bruno Hiroshi Tsuneda1 ORCID - http://orcid.org/0000-0002-7957-2452 \\ Luciana Keiko Hatamoto-Zervoudakis ${ }^{1}$ ORCID - http://orcid.org/0000-0001-6030-096X \\ Joanis Tilemahos Zervoudakis ${ }^{1}$ ORCID - http://orcid.org/0000-0002-1864-3237 \\ Walter Augusto dos Santos Marinho3 ORCID -http://orcid.org/0000-0003-1626-8629 \\ Moacir Ferreira Duarte Júnior ${ }^{1}$ ORCID - http://orcid.org/0000-0001-7383-1102 \\ Eleonora Barbosa Araújo² ORCID - http://orcid.org/0000-0002-3813-5272 \\ Tathiana Ferguson Motheo ${ }^{1}$ ORCID - http://orcid.org/0000-0002-2202-0331 \\ Luis Eduardo Senra e Silva1 ORCID - http://orcid.org/0000-0002-5703-0249 \\ 1Universidade Federal do Mato Grosso, Cuiabá, MT, Brasil. \\ 2EMBRAPA Recursos Genéticos e Biotecnologia, Brasília, DF, Brasil. \\ 3 Universidade Federal do Mato Grosso, Cuiabá, MT, Brasil; Instituto Federal de Ciência e Tecnologia de \\ Mato Grosso, São Vicente, MT, Brasil. \\ *Autor para correspondência - pedrotsuneda@hotmail.com
}

\section{Resumo}

Objetivou-se avaliar a qualidade espermática do sêmen de touros suplementados com selênio (Se) na dieta. Foram utilizados 16 touros Brangus, igualmente distribuídos em grupo controle (GC) e grupo $\mathrm{Se}$ (GSe - 0,1 mg de Se/kg de MS de dieta). O experimento teve duração de 75 dias, e os animais foram suplementados por 60 dias. Foram realizadas quatro coletas de sêmen durante o período $(0,30,60$ e 75 dias) por animal. As amostras foram avaliadas quanto a motilidade e vigor espermáticos, integridade e funcionalidade da membrana plasmática (teste de expansão hiposmótico - HIPO) e viabilidade espermática e reação acrossomal (coloração tripla - TRI). Após avaliação, estas foram diluídas

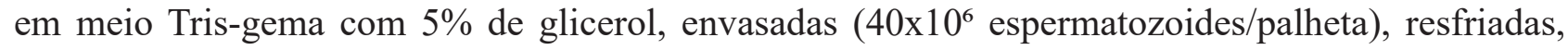
congeladas e armazenadas em nitrogênio líquido até a análise. Após descongelação, foram submetidas às mesmas avaliações descritas para o sêmen fresco. Não houve interferência da suplementação com Se nas variáveis vigor espermático, HIPO e TRI do sêmen fresco e descongelado. Porém, constatouse queda na motilidade espermática do GSe comparativamente ao GC no sêmen fresco $(\mathrm{P}=0,0035) \mathrm{e}$ descongelado $(\mathrm{P}=0,0067)$ após 60 dias de suplementação. Portanto, a suplementação de Se na dieta não foi efetiva na promoção de melhorias dos parâmetros espermáticos de touros Brangus.

Palavras-chave: antioxidantes, criopreservação, espermatozoide.

\begin{abstract}
The aim of the present study was to evaluate sperm quality of bulls supplemented with selenium (Se) in the diet. Sixteen Brangus bulls were randomly divided in two groups: control (GC) and Se (GSe $-0.1 \mathrm{mg} \mathrm{Se} / \mathrm{kg}$ dietary DM). The experiment lasted 75 days and the animals were supplemented for 60 days. Four semen collections (0, 30, 60 and 75 days) per animal, were performed during
\end{abstract}


the experimental period. Sperm motility and vigor, plasma membrane integrity and functionality (hyposmotic swelling test - HOST) and sperm viability and acrosome reaction (triple staining -TRI) were assessed. After immediate analysis, samples were diluted in Tris-egg yolk extender with 5\%

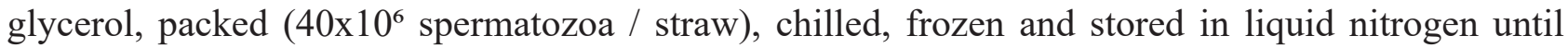
analysis. After thawing, sperm motility and vigor, HOST and TRI were performed. No significance was noticed on sperm vigor, HOST and TRI of fresh and post-thawed semen after dietary selenium supplementation. However, sperm motility decreased in GSe compared to GC in fresh $(\mathrm{P}=0.0035)$ and post- thawed $(\mathrm{P}=0.0067)$ semen samples after 60 days of supplementation. Therefore, dietary selenium supplementation was ineffective to improve semen parameters of fresh and post-thawed semen of Brangus bulls.

Keywords: antioxidant, cryopreservation, spermatozoa.

Recebido em 07 de agosto de 2017.

Aceito em 18 de setembro de 2018.

\section{Introdução}

Dentre as biotecnologias reprodutivas, o processo de criopreservação apresenta contribuição importante no aumento da produção bovina em rebanhos comerciais e de animais de alto valor zootécnico ${ }^{(1)}$. Entretanto, a criopreservação de sêmen é um processo que promove grande estresse celular e impõe aos espermatozoides condições extremamente desfavoráveis à manutenção de sua viabilidade $^{(2)}$

Durante o processo de congelação seminal, a peroxidação lipídica associada ao estresse oxidativo tem sido relacionada à ocorrência de efeitos deletérios na função reprodutiva do macho ${ }^{(3)}$.

A membrana plasmática do espermatozoide é formada por uma bicamada lipídica, que é extremamente vulnerável à ação de espécies reativas ao oxigênio (EROS) ${ }^{(4)}$. Além disso, em bovinos, as EROS podem ser geradas por espermatozoides mortos como resultado de uma reação catalisada oxidativa de aminoácidos ${ }^{(5)}$.

No sêmen, a produção e a degradação das espécies reativas ao oxigênio são consideradas fisiológicas, sendo cruciais em mecanismos responsáveis pela fertilidade espermática. Em contrapartida, o processo de criopreservação aumenta a produção de EROS, a qual resulta em desequilíbrio entre a sua produção e a eliminação pelo sistema antioxidante intra e extracelular ${ }^{(6)}$.

A maioria dos organismos vivos desenvolve complexos sistemas antioxidantes para proteção e reparação de suas células. Estes, por sua vez, atuam por meio da ação de enzimas ou micromoléculas ${ }^{(7)}$ e são capazes de prevenir a formação de EROS, inativar e/ou remover produtos do processo de lipoperoxidação ${ }^{(8)}$ e promover o reparo de lesões promovidas pelas $\operatorname{EROS}^{(9)}$.

Dentre os agentes antioxidantes, destacam-se alguns nutrientes "sequestradores" enzimáticos e algumas enzimas, como a glutationa peroxidase (GPx). Esta, por sua vez, é uma enzima citoplasmática antioxidante abundante no meio intracelular e é frequentemente oxidada em dissulfeto de glutationa ${ }^{(10)}$. O selênio é um elemento essencial para várias funções do organismo, como crescimento, resposta imunológica e manutenção da integridade celular e tecidual. Este atua como cofator da glutationa 
peroxidase, que protege a célula contra o estresse oxidativo ${ }^{(11)}$.

Sendo assim, dada a importância dos danos oxidativos provocados pelo processo de congelação sobre a qualidade espermática de reprodutores bovinos e dos possíveis efeitos benéficos da suplementação com selênio, o objetivo do presente estudo foi avaliar a influência da suplementação da dieta com selênio sobre a qualidade espermática do sêmen de touros Brangus.

\section{Material e métodos}

Todos os procedimentos aqui descritos foram revisados e aprovados pelo Comitê de Ética e BemEstar Animal da Universidade Federal do Mato Grosso (número do protocolo: 23108.060964 / 13-6).

O experimento foi realizado na Fazenda Sereno - GAP, localizada no município de Jaciara (Latitude 15.9561, Longitude $54963215^{\circ}, 57^{\prime} 22^{\prime}$ 'Sul, 54 57’48”Oeste), no período da seca (junho a setembro).

Foram utilizados 16 touros da raça Brangus (5/8 Angus 3/8 Zebu), com idade média de dois anos e peso vivo médio inicial de $472 \mathrm{~kg}$. Previamente ao início do experimento, os animais foram submetidos ao controle de endoparasitas (Doramectina 1\%, Dectomax ${ }^{\circledR}$ Zoetis, Brasil) e ectoparasitas (Cipermetrina e Ethion, Ciperthion ${ }^{\circledR}$, Intervet Schering-Plough, Brasil).

A área experimental destinada aos animais foi constituída por dois piquetes de 7,35/ha cada, com Panicum maximum cv. Mombaça, os quais foram providos de bebedouro e cocho com dimensões que permitiam o acesso de todos os animais simultaneamente.

Os animais foram mantidos em pastejo contínuo, recebendo diariamente suplementação concentrada (Tabela 1).

Tabela 1. Composição percentual do suplemento fornecido na dieta

\begin{tabular}{ll}
\hline Ingrediente & Quantidade (\%) \\
\hline Farelo de soja & 27,50 \\
Milho moído & 67,25 \\
Calcário & 1,50 \\
Enxofre & 0,25 \\
Ureia pecuária & 0,50 \\
Sal mineral $88^{*}$ & 3,00 \\
\hline Niveis de garantia por kg de suplemento mineral: $\mathrm{Ca}-154 \mathrm{~g} ; \mathrm{P}-88 \mathrm{~g} ; \mathrm{Cu}-1700 \mathrm{mg} ; \mathrm{S}-12 \mathrm{~g} ; \mathrm{Co}-200 \mathrm{mg} ; \mathrm{Mn}$ \\
$1300 \mathrm{mg} ; \mathrm{Zn}-5000 \mathrm{mg} ; \mathrm{I}-130 \mathrm{mg} ; \mathrm{Se}-20 \mathrm{mg} ; \mathrm{Mg}-6 \mathrm{mg} ; \mathrm{Na}-130 \mathrm{~g}$.
\end{tabular}

Os suplementos foram formulados de forma a apresentarem $21 \%$ de PB na matéria natural. Estes eram fornecidos em quantidade equivalentes a 4,5 kg/animal/dia, sempre às 11:00, a fim de minimizar as interferências no comportamento de pastejo.

Considerando-se a categoria animal e peso vivo médio dos animais, estimou-se o consumo de matéria seca (CMS) em $11,55 \mathrm{~kg}$ de MS/ dia $^{(12)}$. Foram coletadas amostras de pasto e do suplemento para posterior análise no Laboratório de Nutrição Animal da Faculdade de Agronomia e Zootecnia da 
Universidade Federal de Mato Grosso (FAAZ - UFMT). Dessa forma, realizou-se pré-secagem das amostras em estufa ventilada por 72 horas e, ato contínuo, estas foram processadas em moinho com peneira de malha de 1,0 mm e acondicionadas em potes de plástico. Foram determinados os teores de matéria seca (MS), proteína bruta (PB), fibra em detergente neutro (FDN), extrato etéreo (EE) e matéria mineral $(\mathrm{MM})$. Exceto pela análise de $\mathrm{FDN}^{(13)}$, as demais análises seguiram as recomendações de Silva e Queiroz ${ }^{(14)}$ (Tabela 2).

Tabela 2. Composição químico-bromatológica do suplemento concentrado e da forragem utilizados na dieta

\begin{tabular}{lll}
\hline Nutriente & Concentrado (\%) & Forragem (\%) \\
\hline Matéria seca (MS)* & 90,00 & 37,99 \\
Proteina bruta (PB)** & 21,00 & 6,23 \\
Fibra em detergente neutro (FDN)** & 29,40 & 67,57 \\
Extrato etéreo (EE)** & 2,50 & 1,05 \\
Matéria mineral (MM)** & 8,43 & 7,80 \\
\hline
\end{tabular}

* com base na matéria natural

** com base na matéria seca (MS)

Antes do início do experimento, os animais passaram por um período de adaptação aos piquetes e ao concentrado de 10 dias. Este teve duração de 75 dias, sendo os animais suplementados por 60 dias. Ao final da suplementação, os animais continuaram recebendo o mesmo manejo, sendo fornecido concentrado sem a suplementação de selênio por mais 15 dias para a avaliação de possível efeito residual.

Os animais foram divididos aleatoriamente em dois lotes, os quais foram distribuídos em dois tratamentos: grupo controle (GC, suplemento concentrado) e grupo selênio (GSe, suplemento concentrado com adição de $0,1 \mathrm{mg}$ de $\mathrm{Se} / \mathrm{kg}$ de matéria seca na $\left.\operatorname{dieta}^{(15)}\right)$.

Durante o período experimental, foram realizadas quatro coletas de sêmen, sendo estas realizadas nos dias 0, 30, 60 e 75 (COL 1, COL 2, COL 3 e COL 4, respectivamente). Adotou-se para dia 0 (D0) o dia anterior ao início da suplementação.

Após contenção, os animais foram submetidos a exames clínicos gerais e específicos. Em seguida, o sêmen foi coletado por meio de eletroejaculação, sendo utilizado o aparelho Eletrovet ${ }^{\circledR}$ (Eletro Veterinária Ltda, Valinhos, SP, Brasil). O sêmen foi recolhido em tubo plástico graduado aquecido e protegido da luz. Ato contínuo, foram realizadas análises subjetivas de motilidade ( $0-100 \%$, onde 0\%: nenhum espermatozoide móvel e 100\%: todos os espermatozoides móveis) e vigor espermáticos (0-5, onde 0: ausência de movimento e 5: movimentos vigorosos e progressivos) sob microscopia óptica em aumento de $400 \mathrm{x}^{(16)}$. Dessa forma, uma alíquota de sêmen foi diluída em formol salino na proporção de 1:200 para análise de concentração espermática em câmara de Neubauer16. Ainda, foram avaliadas a integridade e a funcionalidade da membrana plasmática pelo teste de expansão hiposmótico (HIPO) ${ }^{(17)}$ e a integridade da membrana acrossomal e reação acrossomal pela coloração tripla (TRI) ${ }^{(18)}$

Para a realização do teste de expansão hiposmótico, homogeneizaram-se $100 \mu \mathrm{L}$ de amostra a $900 \mu \mathrm{L}$ de solução hiposmótica (150 mOsml). Como controle, o mesmo processo foi realizado, utilizando-se uma solução isosmótica (300 mOsml). As amostras foram então mantidas em banho-maria a $37^{\circ} \mathrm{C}$, 
durante 30 minutos. Transcorrido esse período, uma gota de cada amostra foi colocada entre lâmina e lamínula para a contagem de 200 células, em microscópio de contraste de fase sob aumento de 400x. Os espermatozoides foram classificados quanto a presença (membrana íntegra e funcional) ou não (membrana não íntegra ou afuncional) de cauda enrolada. O resultado foi expresso em porcentagem de células com membrana íntegra e obtido pela diferença entre a porcentagem de células espermáticas com cauda enrolada resultantes do teste hiposmótico da porcentagem de espermatozoides com cauda enrolada patológica (resultantes da solução isosmótica) ${ }^{(17)}$.

A integridade da membrana acrossomal e a reação acrossômica foi avaliada por meio da coloração tripla (TRI). Sendo assim, $300 \mu \mathrm{L}$ de suspensão espermática foram diluídos em igual volume de solução de $0,85 \%$ de $\mathrm{NaCl}$, contendo $2 \%$ de corante "trypan blue". Em seguida, a mistura foi incubada a $37^{\circ} \mathrm{C}$ por 15 minutos e, após esse período, foi lavada em tampão fosfato salino (PBS) por meio de centrifugação a $400 \mathrm{G}$ por 5 minutos. O processo foi continuamente repetido até que o sobrenadante ficasse límpido. Após o descarte do sobrenadante da última lavagem, o pellet formado foi homogeneizado com $50 \mu \mathrm{L}$ de solução de glutaraldeído a $3 \%$ e posteriormente incubado a $36{ }^{\circ} \mathrm{C}$ em banho-maria por 20 minutos. Transcorrido esse período, foi acrescentado o mesmo volume de PBS, e a amostra foi novamente centrifugada a $400 \mathrm{G}$ por 5 minutos. O excesso de sobrenadante foi descartado e foram confeccionados esfregaços com o material remanescente. Após secagem, as lâminas foram coradas em solução de Bismarck Brown $(0,8 \%)$ a $40{ }^{\circ} \mathrm{C}$ por 5 minutos e, em seguida, coradas em solução de Rosa Bengala $(0,8 \%)$ em temperatura ambiente por 20 minutos. O excesso de corante foi lavado em água corrente e as lâminas secas a temperatura ambiente.

Para mensurar a viabilidade e o estado do acrossoma, foram avaliadas 200 células, sob microscopia de contraste de fase em aumento de 1250X. De acordo com a técnica, foram observados quatro padrões de coloração: TRI I - espermatozoides vivo e intactos: corados de castanho na região pósacrossomal e de rosa no acrossomo; TRI II - espermatozoides vivos com reação acrossômica: corados de castanho na região pós-acrossomal e de branco no acrossomo; TRI III-espermatozoides mortos com acrossomo intacto: corados de azul na região pós-acrossomal e de rosa no acrossomo, e TRI IV - espermatozoides mortos com reação acrossômica degenerativa: corados de azul na região pósacrossomal e de branco no acrossomo ${ }^{(18)}$.

Após avaliação imediata, cada ejaculado foi diluído em extensor tris-gema (3,187g TRIS-hidroximetil amino metano; $1,78 \mathrm{~g}$ ácido cítrico monohidratado; $1,316 \mathrm{~g}$ frutose; $80 \mathrm{~mL}$ água destilada; $20 \mathrm{~mL}$ gema de ovo; $100 \mathrm{mg}$ de estreptomicina; 100000UI de penicilina; $5 \mathrm{~mL}$ de glicerol para cada $100 \mathrm{~mL}$ de diluidor) ${ }^{(19)}$ em fração única para obtenção de concentração de 40x10 espermatozoides/palheta. Cinco palhetas de sêmen de cada animal por coleta foram submetidas ao processo de criopreservação de acordo com a metodologia descrita por Beconi et al. ${ }^{(20)}$. Dessa forma, o sêmen foi envasado em palhetas de $0,5 \mathrm{~mL}$, as quais foram submetidas a 4 horas de resfriamento $\left(4{ }^{\circ} \mathrm{C}\right)$. Ato contínuo, estas foram colocadas em contato com o vapor de nitrogênio (a $5 \mathrm{~cm}$ do nitrogênio líquido, $-120^{\circ} \mathrm{C}$ ) por 15 minutos e em seguida submergidas em nitrogênio líquido. Por fim, as amostras foram armazenadas em botijão criogênico $\left(-196^{\circ} \mathrm{C}\right)$ até análise.

A descongelação foi realizada segundo a metodologia proposta por Beconi et al. ${ }^{(20)}$. Sendo assim, as amostras foram descongeladas a $37^{\circ} \mathrm{C}$ durante 30 segundos e $10 \mu \mathrm{L}$ foram utilizados para análise de motilidade e vigor espermáticos. Ainda, foram retiradas alíquotas de sêmen para realização do teste de expansão hiposmótico e para coloração tripla.

$\mathrm{O}$ experimento foi realizado em delineamento inteiramente casualizado com medidas repetidas no tempo. Os dados foram analisados através de análise de variância (ANOVA) com um nível de significância de 5\% com auxílio do programa estatístico SAS (Cary, Carolina do Norte, EUA) ${ }^{(21)}$. 


\section{Resultados e discussão}

Em nosso estudo, foram utilizados 16 touros em repouso sexual, fato este que pode explicar a baixa qualidade do sêmen a fresco observada na primeira coleta (Tabela 3 ) e nos valores obtidos nas demais coletas e pós-criopreservação (Tabela 4).

Ainda, ao longo do período experimental, não foi observada interferência da suplementação com selênio na dieta nos valores de vigor espermático, integridade e funcionalidade da membrana plasmática (HIPO) e integridade da membrana acrossomal e reação acrossomal (TRI) do sêmen fresco e descongelado. Em contrapartida, ao avaliar a motilidade espermática, foi constatado aumento gradativo nos valores dessa variável tanto no grupo controle como no grupo suplementado com selênio. Porém, após 60 dias de suplementação, o grupo suplementado apresentou queda nos valores de motilidade comparativamente ao grupo controle $(\mathrm{P}=0,0035)$ e, após o fim da suplementação com selênio na dieta, a porcentagem de espermatozoides móveis voltou a aumentar (Tabela 3). Comportamento semelhante foi constatado em amostras criopreservadas obtidas da terceira coleta ( $\mathrm{P}=0,0067)$ (Tabela 4), na qual a motilidade espermática observada no grupo suplementado com selênio foi inferior ao grupo não suplementado.

Tabela 3. Média \pm erro-padrão da média de circunferência escrotal $(\mathrm{CE})$, concentração espermática (CO), motilidade (MOT) e vigor (VIG) espermáticos, integridade e funcionalidade da membrana plasmática (HIPO), integridade da membrana acrossomal e reação acrossômica (TRI) do sêmen fresco de touros Brangus com (GSe) e sem a suplementação (GC) com selênio na dieta aos $0,30,60$ e 75 dias

\begin{tabular}{lllllllll}
\hline \multirow{2}{*}{ Parâmetros } & $\mathbf{0}$ & \multicolumn{3}{l}{$\mathbf{3 0}$} & \multicolumn{6}{c}{$\mathbf{6 0}$} & \multicolumn{3}{l}{$\mathbf{7 5}$} \\
\cline { 2 - 8 } & GC & GSe & GC & GSe & GC & GSe & GC & GSe \\
\hline MOT (\%) & $53,3 \pm 5,5$ & $37,5 \pm 8,8$ & $56,1 \pm 6,9$ & $51,3 \pm 8,2$ & $61,7 \pm 4,6^{\mathrm{a}}$ & $36,9 \pm 6^{\mathrm{b}}$ & $66,7 \pm 2,8$ & $54,3 \pm 10,3$ \\
VIG (1-5) & $2,7 \pm 0,2$ & $2 \pm 0,3$ & $2,8 \pm 0.1$ & $2,3 \pm 0,3$ & $2,5 \pm 0,2$ & $2 \pm 0,3$ & $2,7 \pm 0,2$ & $2,3 \pm 0,3$ \\
HIPO (\%) & $43,8 \pm 6,8$ & $42,6 \pm 7,1$ & $17,2 \pm 3,7$ & $28,7 \pm 6,5$ & $34,8 \pm 4,7$ & $46 \pm 9$ & $28,9 \pm 4,6$ & $37,6 \pm 3$ \\
TRI I (\%) & - & - & $91,1 \pm 2,1$ & $88,7 \pm 3$ & $89,4 \pm 2,7$ & $82,6 \pm 6,8$ & $95 \pm 1$ & $96 \pm 1,9$ \\
TRI II (\%) & - & - & $0,4 \pm 0,1$ & $0,8 \pm 0,34$ & $0,8 \pm 0,3$ & $0,3 \pm 0,1$ & $0,4 \pm 0,1$ & $0,7 \pm 0,1$ \\
TRI III (\%) & - & - & $7,6 \pm 2,1$ & $9,4 \pm 2,5$ & $5,6 \pm 1,3$ & $14,1 \pm 6,1$ & $3,5 \pm 0,8$ & $1,6 \pm 0,7$ \\
TRI IV (\%) & - & - & $0,8 \pm 0,5$ & $1,1 \pm 0,6$ & $4,4 \pm 1,8$ & $2,9 \pm 0,9$ & $1 \pm 0,3$ & $1,7 \pm 1,3$ \\
\hline
\end{tabular}

${ }^{a, b}$ Letras sobrescritas na mesma linha indicam diferença significativa entre os tratamentos.

$\mathrm{P} \leq 0,05$

Inúmeros estudos associam a suplementação com selênio à performance reprodutiva de machos e a melhorias na concentração, motilidade e morfologia espermáticas (22 - 26). Tais benefícios estão associados principalmente a sua ação como cofator da enzima glutationa peroxidase que, por sua vez, atua neutralizando a ação das EROS sobre o espermatozoide (10 - 11, 20, 22-23). Porém, no presente estudo, apesar da utilização de concentrações de selênio recomendadas para essa categoria animal, sua utilização contínua na dieta foi deletéria para a motilidade espermática, sugerindo possível efeito acumulativo e tóxico desse mineral, uma vez que, após sua remoção, constatou-se aumento dessa variável. 
Tabela 4. Média \pm erro-padrão da média de motilidade (MOT) e vigor (VIG) espermáticos, integridade e funcionalidade da membrana plasmática (HIPO) e integridade da membrana acrossomal (TRI) e reação acrossômica do sêmen descongelado de touros Brangus com (GSe) e sem a suplementação (GC) com selênio na dieta aos $0,30,60$ e 75 dias

\begin{tabular}{lllllllll}
\hline \multirow{2}{*}{ Parâmetros } & $\mathbf{0}$ & \multicolumn{3}{c}{$\mathbf{3 0}$} & $\mathbf{6 0}$ & \multicolumn{2}{c}{$\mathbf{7 5}$} & \\
\cline { 2 - 8 } & GC & GSe & GC & GSe & GC & GSe & GC & GSe \\
\hline MOT (\%) & $6,3 \pm 0,8$ & $4,3 \pm 2$ & $24,4 \pm 6,2$ & $27,1 \pm 8,2$ & $17,8 \pm 4,9^{\mathrm{a}}$ & $5 \pm 0,9^{\mathrm{b}}$ & $2.5 \pm 1,1$ & $12,1 \pm 4,7$ \\
VIG (1-5) & $1,4 \pm 0,2$ & $0,7 \pm 0,3$ & $2,5 \pm 0,4$ & $2,3 \pm 0,5$ & $2,4 \pm 0,2$ & $1,7 \pm 0,4$ & $0,7 \pm 0,3$ & $1,3 \pm 0,3$ \\
HIPO (\%) & $10,8 \pm 4,1$ & $9,7 \pm 2,2$ & $11 \pm 2,7$ & $9,2 \pm 1,5$ & $6,9 \pm 1,7$ & $12,8 \pm 3,6$ & $8,4 \pm 3,3$ & $7,1 \pm 2,1$ \\
TRI I(\%) & $4,4 \pm 2,3$ & $5,5 \pm 2,8$ & $14,3 \pm 6,4$ & $3,3 \pm 1,7$ & $15,7 \pm 5,7$ & $9,3 \pm 7$ & $15,1 \pm 10,5$ & $15,1 \pm 4$ \\
TRI II (\%) & 0 & $0,1 \pm 0,1$ & $0,2 \pm 0,1$ & 0 & $0,1 \pm 0,1$ & 0 & 0 & $0,2 \pm 0,1$ \\
TRI III (\%) & $93,1 \pm 2,3$ & $91,6 \pm 2,4$ & $84,3 \pm 6,3$ & $95,7 \pm 1,6$ & $82,9 \pm 5,7$ & $89,3 \pm 7,1$ & $83,1 \pm 10,6$ & $83,2 \pm 4,1$ \\
TRI IV (\%) & $2,4 \pm 0,3$ & $2,8 \pm 0,6$ & $1,21 \pm 0,3$ & $1 \pm 0,3$ & $1,2 \pm 0,2$ & $1,4 \pm 0,2$ & $1,7 \pm 0,2$ & $1,5 \pm 0,1$ \\
\hline
\end{tabular}

${ }^{2, b}$ Letras sobrescritas na mesma linha indicam diferença significativa entre os tratamentos.

$\mathrm{P} \leq 0,05$

A ação de qualquer antioxidante sobre a fertilidade do sêmen respeita uma regressão polinomial, ou seja, à medida que se adiciona a substância com propriedade de neutralizar os agentes oxidantes, espera-se que a variável relacionada à fertilidade dos espermatozoides aumente, principalmente se o sistema já estiver em desequilíbrio. Contudo, aos poucos a adição de antioxidantes passa a deprimir a fertilidade, isso porque sabe-se que as espécies reativas de oxigênio participam de diversas reações da célula espermática, sendo que várias delas afetam os padrões de movimento ${ }^{(27)}$. Desse modo, o uso prolongado do selênio na dieta pode ter afetado negativamente a motilidade do sêmen fresco e, consequentemente, do sêmen criopreservado.

Embora seja pouco descrito, o selênio proveniente da dieta pode levar a quadros de intoxicação. Compostos derivados do selênio como selenito, disselenito e dióxido de selênio podem reagir com tióis e produzir superóxido e outras espécies reativas de oxigênio capazes de causar danos aos espermatozoides $^{(28)}$. Ainda, apesar de alguns autores relatarem deficiência de selênio nas pastagens ${ }^{(23)}$, acredita-se que a dosagem desse elemento é essencial para que não ocorra uma suplementação excessiva desse mineral de animais mantidos a pasto. Dessa forma, apesar da ausência de sinais clínicos de toxicidade, o decréscimo da motilidade espermática constatado em nosso estudo pode ter sido decorrente de excesso de selênio na dieta, uma vez que os animais eram mantidos a pasto e a suplementação foi feita com base no $\mathrm{NRC}^{(12)}$ específico para essa categoria animal.

Trabalhos que relacionem o efeito da suplementação com selênio com os parâmetros qualitativos e quantitativos do sêmen de bovinos são raros. Sendo assim, mais estudos na área, variando os níveis e a fonte do selênio, se fazem necessários para elucidar o efeito desse mineral na atividade reprodutiva desses animais.

\section{Conclusão}

Com base nos resultados encontrados, conclui-se que a suplementação com selênio na concentração 
de $0,1 \mathrm{mg} / \mathrm{kg}$ de MS na dieta não foi efetiva na promoção de melhorias na qualidade espermática do sêmen fresco e criopreservado de touros da raça Brangus.

\section{Referências}

1. Leite PA, Schreder GG, Almeida CLR, Zúccari CESN, Costa e Silva EV. Criopreservação do sêmen bovino. Cientifica Ciências Biologia e Saúde. 2011; 13(4):279-86.

2. Purdy PH. A review on goat sperm cryopreservation. Small Rum Res. 2006; 63:215-225. Doi:10.1016/j. smallrumres.2005.02.015

3. Aitken RJ, Jones KT, Robertson SA. Reactive oxigens species and sperm function - in sickness and in health. J Androl. 2012; 33:1096-1106. Doi:10.2164/jandrol.112.016535.

4. Brouwers JFHM, Gadella BM. In situ detection and localization of lipid peroxidation in individual bovine sperm cells. Free Rad Biol Med. 2003; 35:1382-1391. Doi: 10.1016/j.freeradbiomed.2003.08.010

5. Sariözkan S, Bucak MN, Tuncer PB, Ulutas PA, Bilgen A. The influence of cysteine and taurine onmicroscopic-oxidative stress parameters and fertilizing ability of bull semen following cryopreservation. Cryobiology. 2009; 58:134-138.Doi: 10.1016/j.cryobiol.2008.11.006

6. Maia MS, Bicudo SD. Radicais livres, antioxidantes e função espermática em mamíferos: uma revisão. Rev Bra Reprod Anim. 2009; 33(4):183-193.

7. Barreiros ALBS, David JM, David JP. Estresse oxidativo: relação entre geração de espécies reativas e defesa do organismo. Quím Nova. 2006; 29(1):113-123. Doi:10.1590/S0100-40422006000100021

8. Huang Z, Roze AH, Hoffmann PR. The role of selenium inflammation and immunity: from molecular mechanisms to therapeutic opportunities. Antiox Redox Signal. 2012; 16(7):705-743. Doi:10.1089/ $\underline{\operatorname{ars} .2011 .4145}$

9. Bianchi ML, Antunes LMG. Radicais livres e os principais antioxidantes da dieta. Rev Nutr, 1999; 12,(2):123-130. Doi:10.1590/S1415-52731999000200001

10. Yoshihara D, Fujiwara N, Ookawara T, Kato S, Sakiyama H, Yokoe S, Eguchi H, Suzuki K. Protective role of glutathione S-transferase A4 induced in copper/zinc-superoxide dismutase knockout mice. Free Radic Biol Med. 2009; 47:559-567. Doi: 10.1016/j.freeradbiomed.2009.05.022

11. Alvarez CA, Moraes GV. Efeitos da selenometionina e vitamina c sobre o sêmen. SaBios: Revista de Saúde e Biologia. 2006; 1(1):42-51.

12. National Academies of Sciences, Engineering, and Medicine. 2016. Nutrient Requirements of Beef Cattle, Eighth Revised Edition. Washington, DC: The National Academies Press.

13. Van Soest PJ, Robertson JB, Lewis BA. Methods for dietary fiber, and nonstarch polysaccharides in relation to animal nutrition. J Dairy Sci. 1991; 74 (10): 3583-3597. Doi:10.3168/jds.S0022-0302(91)78551-2

14. Silva DJ, Queiroz AC. Análise de alimentos: métodos químicos e biológicos. 3.ed. Viçosa, MG: Universidade Federal de Viçosa, 2002. 165p.

15. Suttle, NF. Mineral Nutrition of livestock, 4.ed. Oxfordshire, UK: Cabi, 2010. 579p. 
16. CBRA. Colégio Brasileiro de Reprodução Animal. Manual para exame andrológico e avaliação de sêmen animal. 3. ed. Belo Horizonte, 2013.

17. Jeyendran RS, Van der vem HH, Perez-Pelaez M, Gravo BG, Zaneveld LJD. Development of an assay to assess the functional integrity of the human sperm membrane and its relationship to other semen characteristics. J Reprod Fertil. 1984; 70(1): 219-228.

18. Harayama H, Kusunoki H, Kato S. Capacity of goat epididymal spermatozoa to undergo the acrosome reaction and subsequent fusion with the egg plasma membrane. Reprod Fertil Dev.1993; 5(3):239-246.

19. Rota A, Strom B, Linde-Forseberg C. Effects of seminal plasma and three extenders on canine semen stored at $4{ }^{\circ} \mathrm{C}$. Theriogenology, $1995 ; 44: 885-900$.

20. Beconi MT, Affranchino MA, Schang LM, Beorlegui NB. Influence of antioxidants on SOD activity in bovine sperm. Biochem Int. 1991; 23(3):545-553.

21. SAS. 2001. The statistical analyse system for windows: version 8. Cary, 1999-2001. CD-Rom.

22. Sergerson EC, Johnson BH. Selenium and reproductive function in yearling Angus bulls. J Anim Sci. 1980; $51: 395-401$.

23. Pasa C. Relação reprodução animal e os minerais. Rev Biod. 2010; 9(1): 101 -122.

24. Bartle JL, Senger PL, Hillers JK. Influence of injected selenium in dairy bulls on blood and semen selenium, glutathione peroxidase and seminal quality. Biol Reprod. 1980; 23:1007-1013.

25.Ahsan U, Kamran Z, Raza I, Ahmad S, Babar W, Riaz MH, Iqbal Z. Role of selenium in male reproduction-A review:SLẸ. Anim Reprod Sci. 2014; 146: 55-62. Doi:10.1016/j.anireprosci.2014.01.009

26. Marai IFM, El-Darawany AA, Ismail EA, Abdel-Hafez MAM. Reproductive and physiological traits of Egyptian Suffolk rams as affected by selenium dietary supplementation and housing heat radi- ation effects during winter of the sub-tropical environment of Egypt. Arch. Tierz. 2009; 52: 402-409. Doi:10.1016/j. anireprosci.2014.01.009

27. Domosławska A, Zdunczyk S, Franczyk M, Kankofer M, Janowski T. Selenium and vitamin E supplementation enhances the antioxidant status of spermatozoa and improves semen quality in male dogs with lowered fertility. Andrologia. 2018;e13023. Doi:10.1111/and.13023

28. Spallholz JE. Free radical generation by selenium compounds and their prooxidant toxicity. Biomed Envirom Sci. 1997; 10:260-270. 